\title{
OPEN A hospital-based study on etiology and prognosis of bacterial meningitis in adults
}

\begin{abstract}
Jun-Sang Sunwoo ${ }^{1}$, Hye-Rim Shin ${ }^{2}$, Han Sang Lee ${ }^{3}$, Jangsup Moon ${ }^{3,4}$, Soon-Tae Lee ${ }^{3}$, Keun-Hwa Jung ${ }^{3}$, Kyung-Il Park ${ }^{5}$, Ki-Young Jung ${ }^{3}$, Manho Kim ${ }^{3,6}$, Sang Kun Lee ${ }^{3}$ \& Kon Chu ${ }^{3 凶}$

Bacterial meningitis is a neurological emergency with high morbidity and mortality. We herein investigated clinical features, etiology, antimicrobial susceptibility profiles, and prognosis of bacterial meningitis in adults from a single tertiary center. We retrospectively reviewed medical records of patients with laboratory-confirmed bacterial meningitis from 2007 to 2016. Patients with recent neurosurgery, head trauma, or indwelling neurosurgical devices were classified as having healthcarerelated meningitis. Causative microorganisms were identified by analyzing cerebrospinal fluid (CSF) and blood cultures, and antimicrobial susceptibility profiles were evaluated. We performed multiple logistic regression analysis to identify factors associated with unfavorable outcomes. We identified 161 cases (age, $55.9 \pm 15.5$ years; male, 50.9\%), of which 43 had community-acquired and 118 had healthcare-related meningitis. CSF and blood culture positivity rates were $91.3 \%$ and $30.4 \%$, respectively. In community-acquired meningitis patients, Klebsiella pneumoniae (25.6\%) was the most common isolate, followed by Streptococcus pneumoniae (18.6\%) and Listeria monocytogenes (11.6\%). The susceptibility rates of $K$. pneumoniae to ceftriaxone, cefepime, and meropenem were $85.7 \%$, $81.3 \%$, and $100 \%$, respectively. Among healthcare-related meningitis patients, the most common bacterial isolates were coagulase-negative staphylococci (28.0\%), followed by Staphylococcus aureus (16.1\%) and Enterobacter spp. (13.6\%). Neurological complications occurred in $39.1 \%$ of the patients and the 3-month mortality rate was $\mathbf{1 4 . 8 \%}$. After adjusting for covariates, unfavorable outcome was significantly associated with old age (odds ratio [OR] 1.03, 95\% confidence interval $[\mathrm{Cl}] 1.00-1.06$ ), neurological complications (OR 4.53, 95\% Cl 1.57-13.05), and initial Glasgow coma scale $\leq 8$ (OR 19.71, $95 \% \mathrm{Cl}$ 4.35-89.40). Understanding bacterial pathogens and their antibiotic susceptibility may help optimize antimicrobial therapy in adult bacterial meningitis.
\end{abstract}

Bacterial meningitis is a neurological emergency with high morbidity and mortality. Over 1.2 million cases of bacterial meningitis are estimated to occur annually worldwide ${ }^{1}$. Although adjunctive dexamethasone reduces the risk of unfavorable outcomes and death, neurological complications occur in approximately $30 \%$ of survivors ${ }^{2,3}$. Delayed antibiotic administration has been shown to significantly increase mortality and adverse outcomes at 3 months ${ }^{4}$. Therefore, early clinical suspicion and immediate antibiotic therapy are crucial in the initial management of bacterial meningitis.

Antibiotic treatments are determined empirically, based on the common causative pathogens of bacterial meningitis, age, host immune status, and predisposing conditions ${ }^{5}$. According to previous epidemiological studies, Streptococcus pneumoniae, Neisseria meningitidis, Haemophilus influenzae, and Listeria monocytogenes are the major bacterial pathogens responsible for community-acquired meningitis in adults ${ }^{6,7}$. On the other hand, the most common microorganisms associated with neurosurgical procedures and head trauma were coagulasenegative staphylococci (CoNS), Staphylococcus aureus, and gram-negative bacilli ${ }^{8}$. However, the epidemiology of bacterial meningitis has changed over the past 30 years. The introduction of conjugate vaccines significantly decreased the incidence of $H$. influenzae and $S$. pneumoniae meningitis and also shifted the age distribution of

\footnotetext{
${ }^{1}$ Department of Neurosurgery, Seoul National University Hospital, Seoul, South Korea. ${ }^{2}$ Department of Neurology, Dankook University Hospital, Cheonan, South Korea. ${ }^{3}$ Department of Neurology, Comprehensive Epilepsy Center, Biomedical Research Institute, Seoul National University Hospital, 101, Daehak-ro, Jongno-gu, Seoul 03080, South Korea. ${ }^{4}$ Rare Disease Center, Seoul National University Hospital, Seoul, South Korea. ${ }^{5}$ Department of Neurology, Seoul National University Hospital Healthcare System Gangnam Center, Seoul, South Korea. ${ }^{6}$ Protein Metabolism and Neuroscience Dementia Medical Research Center, Seoul National University College of Medicine, Seoul, South Korea. ${ }^{\square}$ email: stemcell.snu@gmail.com
} 
bacterial meningitis from children to older adults ${ }^{9,10}$. The increasing rate of antimicrobial resistance in S. pneumoniae is another important epidemiological trend that should be considered when selecting the appropriate antibiotic therapy ${ }^{11}$. However, there is little information on the etiology and antimicrobial susceptibility profiles of recent bacterial meningitis cases, especially in Korea. Therefore, we investigated the clinical, laboratory, and microbiological profiles of adult bacterial meningitis patients from a single tertiary center over a 10 -year period.

\section{Methods}

Study subjects. We retrospectively reviewed the medical records of adult patients with laboratory-confirmed bacterial meningitis, who were treated in Seoul National University Hospital from 2007 to 2016. Bacterial meningitis was defined as follows according to the World Health Organization recommendation ${ }^{12}$. Suspected cases were defined as any person with clinical features of bacterial meningitis, such as fever, altered consciousness, and meningeal signs. Probable cases were defined as any suspected cases with cerebrospinal fluid (CSF) white blood cell (WBC) count $>100$ cells $/ \mathrm{mm}^{3}$, or CSF WBC count of $10-100$ cells $/ \mathrm{mm}^{3}$ with either protein level $>100 \mathrm{mg} / \mathrm{dL}$ or glucose level $<40 \mathrm{mg} / \mathrm{dL}$. Finally, laboratory-confirmed cases were defined as any suspected or probable cases in which bacterial pathogens were identified in CSF or blood cultures or bacterial antigen detection by CSF latex agglutination test. Only laboratory-confirmed bacterial meningitis cases were selected as study subjects and included in the analysis. Patients with tuberculous meningitis and meningoencephalitis due to non-bacterial pathogens were excluded from the study. When CSF and blood cultures showed discordant results, the isolate from the CSF culture was considered as the causative organism.

Bacterial meningitis cases were classified into community-acquired and healthcare-related meningitis, because they have a different spectrum of bacterial pathogens. Patients with recent neurosurgery (within 1 month of the onset of meningitis); head trauma; or indwelling neurosurgical devices, such as ventriculoperitoneal shunt, extraventricular drain, and lumbar drain, were classified as having healthcare-related meningitis. Patients without evidence of healthcare-related infection were classified as having community-acquired meningitis.

Clinical information. We analyzed demographic information, symptoms and signs at presentation, premorbid functional status, immunocompromised status, concurrent infection, indwelling neurosurgical devices, and recent neurosurgery or head trauma. Severe mental deterioration at admission was defined as an initial Glasgow Coma Scale (GCS) score $\leq 8$. We evaluated the initial CSF profiles, including cell count with differential and protein and glucose levels. Clinical outcomes were measured using a modified Rankin Scale (mRS) score at discharge and 3 months after discharge. An unfavorable outcome was defined as an mRS score $\geq 4$ at 3 months.

Antimicrobial susceptibility test. Antimicrobial susceptibility tests were performed and interpreted according to the Clinical and Laboratory Standards Institute. The results were reported as susceptible, intermediate, or resistant. A bacterial isolate was classified as non-susceptible to an antimicrobial agent when it tested as intermediate or resistant. Multi-drug resistance (MDR) was defined, according to the guidelines of the European Centre for Disease Prevention and Control ${ }^{13}$, as non-susceptibility to at least one agent in three or more antimicrobial categories. In particular, MDR of Streptococcus spp. was defined as non-susceptibility to penicillin and antimicrobials in two or more other non- $\beta$-lactam classes $^{14}$.

Ethical statement. This study protocol was approved by the institutional review board (IRB) of Seoul National University Hospital (No. C-1705-016-851) and was performed in accordance with the principles of the Declaration of Helsinki. Because this was a retrospective medical chart review study, informed consent was not obtained from the participants and the IRB of Seoul National University Hospital granted a waiver of informed consent. All information gathered in this study was anonymized to preserve the participants' privacy.

Statistical analysis. We performed a Student's t-test or a Pearson's chi-square test for between-group comparisons of continuous and categorical variables, respectively. Data that were not normally distributed are presented as median (interquartile range) and were analyzed using a Wilcoxon rank-sum test. We performed multivariate logistic regression analyses to identify factors related to clinical outcomes in bacterial meningitis patients. Dependent variables were unfavorable outcome and mortality at 3 months, analyzed separately. Patients with a premorbid disability, defined as a premorbid mRS score $\geq 3$, were excluded from the analysis. Variables with $P<0.1$ in univariate logistic analyses were included as independent variables. In addition, age, sex, and type of meningitis (healthcare-related vs community-acquired) were included as covariates. A two-tailed $P$-value $<0.05$ was considered statistically significant and statistical analyses were performed using SPSS version 25 (IBM Corp. Armonk, NY, USA).

\section{Results}

Clinical presentation. We identified 161 cases of which 43 had community-acquired meningitis and 118 had healthcare-related meningitis. Six patients, all of whom had a healthcare-related infection, experienced a second episode of bacterial meningitis. Of these, postoperative CSF leak occurred in one patient and intraventricular devices were implanted in the other five patients. Overall, the mean age was $55.9 \pm 15.5$ years and $50.9 \%$ of patients were male (Table 1). There was no significant seasonal variation $(P=0.806)$. Regarding predisposing factors, immunocompromised conditions were present in $31(19.3 \%)$ patients and a concurrent infection, such as pneumonia, catheter-related blood stream infection, or peritonitis, was found in 44 (27.3\%) patients. Among the healthcare-related meningitis patients, $64.4 \%$ underwent recent neurosurgery and $62.7 \%$ had indwelling neurosurgical devices. At presentation, the classic triad of fever, neck stiffness, and altered mental status was 


\begin{tabular}{|c|c|c|c|c|}
\hline Variables & $\begin{array}{l}\text { Total } \\
(n=161)\end{array}$ & $\begin{array}{l}\text { Community-acquired } \\
(n=43)\end{array}$ & $\begin{array}{l}\text { Healthcare-related } \\
(\mathrm{n}=118)\end{array}$ & $P$ \\
\hline Age, years & $55.9 \pm 15.5$ & $64.6 \pm 12.5$ & $52.7 \pm 15.3$ & $<0.001$ \\
\hline Male & $50.9 \%$ & $60.5 \%$ & $47.5 \%$ & 0.144 \\
\hline Body temperature, ${ }^{\circ} \mathrm{C}$ & $37.6 \pm 1.0$ & $37.6 \pm 1.1$ & $37.6 \pm 1.0$ & 0.948 \\
\hline Body temperature $\geq 38^{\circ} \mathrm{C}$ & $38.0 \%$ & $37.2 \%$ & $38.3 \%$ & 0.904 \\
\hline Fever by history & $88.2 \%$ & $93.0 \%$ & $86.4 \%$ & 0.252 \\
\hline Neck stiffness* & $62.5 \%$ & $73.7 \%$ & $52.4 \%$ & 0.049 \\
\hline Mental status change & $52.2 \%$ & $74.4 \%$ & $44.1 \%$ & 0.001 \\
\hline GCS score & $12.3 \pm 3.8$ & $11.2 \pm 4.0$ & $12.7 \pm 3.7$ & 0.032 \\
\hline GCS score $\leq 8$ & $20.5 \%$ & $30.2 \%$ & $16.9 \%$ & 0.065 \\
\hline Symptom triad ${ }^{*}$ & $31.3 \%$ & $47.4 \%$ & $16.7 \%$ & 0.003 \\
\hline Systolic BP, mmHg & $134.5 \pm 26.1$ & $142.4 \pm 29.8$ & $131.6 \pm 24.1$ & 0.037 \\
\hline Diastolic BP, mmHg & $79.4 \pm 16.0$ & $81.3 \pm 13.9$ & $78.7 \pm 16.7$ & 0.373 \\
\hline \multicolumn{5}{|l|}{ Predisposing factors } \\
\hline Immunocompromised & $19.3 \%$ & $11.6 \%$ & $22.0 \%$ & 0.138 \\
\hline Concurrent infections & $27.3 \%$ & $14.0 \%$ & $32.2 \%$ & 0.027 \\
\hline Diabetes mellitus & $17.4 \%$ & $27.9 \%$ & $13.6 \%$ & 0.034 \\
\hline Recent neurosurgery $\dagger$ & $47.2 \%$ & 0 & $64.4 \%$ & $<0.001$ \\
\hline Neurosurgical devices & $46.0 \%$ & 0 & $62.7 \%$ & $<0.001$ \\
\hline CSF leakage & $7.5 \%$ & $2.3 \%$ & $9.3 \%$ & 0.184 \\
\hline
\end{tabular}

Table 1. Clinical features of bacterial meningitis at presentation. Data are presented as mean \pm SD or $\%$. ${ }^{\star}$ Data were available in 85 cases. $†$ Recent neurosurgery refers to neurosurgery within 1 month of the onset of meningitis. Abbreviations: GCS, Glasgow coma scale; CSF, cerebrospinal fluid.

\begin{tabular}{|c|c|c|c|c|}
\hline Variables & $\begin{array}{l}\text { Total } \\
(n=161)\end{array}$ & Community-acquired $(n=43)$ & $\begin{array}{l}\text { Healthcare-related } \\
(\mathrm{n}=118)\end{array}$ & $P$ \\
\hline \multicolumn{5}{|l|}{ CSF profiles } \\
\hline Pressure, $\mathrm{cmH}_{2} \mathrm{O}$ & $22.5 \pm 10.6$ & $22.9 \pm 8.9$ & $22.1 \pm 12.3$ & 0.753 \\
\hline WBC count, $/ \mathrm{mm}^{3}$ & $828.0(256.3-2870.0)$ & $1215.0(234.0-4464.0)$ & $800.0(257.0-2690.0)$ & 0.394 \\
\hline$\leq 99 / \mathrm{mm}^{3}$ & $12.5 \%$ & $11.6 \%$ & $12.8 \%$ & \\
\hline $100-999 / \mathrm{mm}^{3}$ & $78.8 \%$ & $76.7 \%$ & $79.5 \%$ & \\
\hline$\geq 1000 / \mathrm{mm}^{3}$ & $8.8 \%$ & $11.6 \%$ & $7.7 \%$ & \\
\hline Neutrophils, \%† & $76.3 \pm 26.1$ & $80.6 \pm 26.0$ & $74.8 \pm 26.1$ & 0.222 \\
\hline Protein, $\mathrm{mg} / \mathrm{dL}$ & $201.8(93.0-489.0)$ & $332.0(165.0-523.0)$ & $177.0(82.8-405.9)$ & 0.005 \\
\hline Glucose, mg/dL & $44.0(10.0-68.0)$ & $21.0(5.0-52.0)$ & $51.0(20.0-69.3)$ & 0.002 \\
\hline Glucose ratio (CSF/blood), $\%^{*}$ & $27.8(6.8-47.3)$ & $14.9(4.0-28.7)$ & $34.2(11.6-51.2)$ & $<0.001$ \\
\hline \multicolumn{5}{|l|}{ Blood tests $\dagger$} \\
\hline Platelet count, $\times 10^{3} / \mathrm{mm}^{3}$ & $218.7 \pm 104.1$ & $159.9 \pm 92.3$ & $240.5 \pm 100.0$ & $<0.001$ \\
\hline hs-CRP, mg/dL & $10.8 \pm 10.9$ & $15.3 \pm 13.7$ & $9.1 \pm 9.1$ & 0.009 \\
\hline
\end{tabular}

Table 2. Cerebrospinal fluid and blood test findings. Data are presented as mean $\pm S D$, median (interquartile range), or $\%$. ${ }^{\star}$ Data were available in 156 cases. $†$ Data were available in 159 cases. Abbreviations: CSF, cerebrospinal fluid; WBC, white blood cell; hs-CRP, high-sensitivity C-reactive protein.

found in $31.3 \%$ of patients. The initial GCS score was $12.3 \pm 3.8$ and severe mental deterioration was observed in $33(20.5 \%)$ patients. Compared to healthcare-related patients, community-acquired meningitis patients were characterized by older age $(P<0.001)$, lower initial GCS scores $(P=0.032)$, and a higher rate of neck stiffness $(P=0.049)$ and the classic symptom triad $(P=0.003)$. Regarding predisposing conditions, patients with healthcare-related meningitis showed a higher prevalence of concurrent infections $(P=0.027)$ and a lower prevalence of diabetes mellitus $(P=0.034)$ than those with community-acquired meningitis.

Laboratory findings. Mean CSF opening pressure was $22.5 \pm 10.6 \mathrm{cmH}_{2} \mathrm{O}$, and an elevated pressure $\geq 20$ $\mathrm{cmH}_{2} \mathrm{O}$ was found in $54.3 \%$ of the patients (Table 2). Median CSF WBC count was $828.0 / \mathrm{mm}^{3}$ (interquartile range [IQR], 256.3-2870.0), with 76.3\% neutrophils and $14.0 \%$ lymphocytes. The median protein level was $201.8 \mathrm{mg} / \mathrm{dL}$ (IQR, 93.0-489.0) and CSF/blood glucose ratio was 0.28 (IQR, 0.07-0.47). Community-acquired meningitis patients showed higher CSF protein levels $(P=0.005)$ and lower CSF glucose levels $(P=0.002)$ than 


\begin{tabular}{|c|c|c|c|}
\hline Microorganisms & Community-acquired $(n=43)$ & Healthcare-related $(\mathrm{n}=118)$ & $P$ \\
\hline \multicolumn{4}{|l|}{ Gram positive } \\
\hline Streptococcus spp. & $34.9 \%$ & $4.2 \%$ & $<0.001$ \\
\hline S. pneumoniae & $18.6 \%$ & $1.7 \%$ & \\
\hline Viridans streptococci & $7.0 \%$ & $2.5 \%$ & \\
\hline S. agalactiae & $9.3 \%$ & 0 & \\
\hline Staphylococcus spp. & $4.7 \%$ & $44.1 \%$ & $<0.001$ \\
\hline S. aureus & $4.7 \%$ & $16.1 \%$ & \\
\hline S. epidermidis & 0 & $17.8 \%$ & \\
\hline Other CoNS & 0 & $10.2 \%$ & \\
\hline Other gram positive & $25.6 \%$ & $11.9 \%$ & 0.033 \\
\hline $\begin{array}{l}\text { Listeria monocy- } \\
\text { togenes }\end{array}$ & $11.6 \%$ & 0 & \\
\hline Enterococcus spp.* & $2.3 \%$ & $6.8 \%$ & \\
\hline Bacillus spp. & $4.7 \%$ & $5.1 \%$ & \\
\hline Others $\dagger$ & $7.0 \%$ & 0 & \\
\hline Gram negative & $34.9 \%$ & $43.2 \%$ & 0.341 \\
\hline Klebsiella pneumoniae & $25.6 \%$ & $5.9 \%$ & \\
\hline Pseudomonas spp. $\neq$ & $7.0 \%$ & $6.8 \%$ & \\
\hline Acinetobacter spp.ll & $2.3 \%$ & $6.8 \%$ & \\
\hline Enterobacter spp.g & 0 & $13.6 \%$ & \\
\hline Others ${ }^{\star *}$ & 0 & $10.2 \%$ & \\
\hline
\end{tabular}

Table 3. Causative microorganisms. Abbreviations: CoNS, Coagulase-negative staphylococci. ${ }^{\star}$ E. faecalis $(\mathrm{n}=5)$ and E. faecium $(\mathrm{n}=4)$. $\dagger$ Corynebacterium species, Micrococcus species, and Parvimonas micra $(\mathrm{n}=1$ for all). $\ddagger$ P. aeruginosa $(\mathrm{n}=10)$ and P. fluorescens $(\mathrm{n}=1)$. $\| A$. baumannii $(\mathrm{n}=7)$ and $A$. lwoffii $(\mathrm{n}=2)$. $g E$. aerogenes $(\mathrm{n}=13)$ and E. cloacae $(\mathrm{n}=3){ }^{* *}$ Serratia marcescenes $(\mathrm{n}=4)$, Stenotrophomonas maltophilia $(\mathrm{n}=3)$, Haemophilus influenzae $(\mathrm{n}=2)$, Escherichia coli $(\mathrm{n}=1)$, Morganella morganii $(\mathrm{n}=1)$, and Moraxella species $(\mathrm{n}=1)$.

healthcare-related meningitis patients, although CSF WBC counts did not significantly differ between the two groups. In blood tests, thrombocytopenia, with a platelet count $<100,000 / \mathrm{mm}^{3}$, was noted in $12.6 \%$ of the patients, whereas increased high-sensitivity C-reactive protein (hs-CRP) levels $>10 \mathrm{mg} / \mathrm{dL}$ were identified in $42.8 \%$ of the patients.

Causative microorganisms. Overall, CSF Gram stains and cultures were positive in 24.5\% (34/139) and $91.3 \%$ (147/161) of patients, respectively. Blood cultures were positive in $30.4 \%$ (49/161) of patients and blood and CSF cultures were both positive in $21.7 \%(35 / 161)$ of patients. Bacterial antigen detection tests were only performed in 40 patients and six of these (15.0\%) were positive, with five testing positive for S. pneumoniae and one testing positive for Streptococcus agalactiae.

The bacterial pathogens isolated from 161 bacterial meningitis cases are summarized in Table 3. Mixed infections with two different species were found in four patients. In community-acquired meningitis patients, Klebsiella pneumoniae (25.6\%) was the most common isolate, followed by S. pneumoniae (18.6\%) and L. monocytogenes (11.6\%). H. influenzae accounted for $4.7 \%$ of infections, but N. meningitidis was not detected. Among healthcare-related meningitis patients, the most common bacterial isolates were CoNS (28.0\%), followed by $S$. aureus (16.1\%) and Enterobacter spp. (13.6\%). Streptococcus spp. were more common in community-acquired meningitis patients $(34.9 \%$ vs. $4.2 \%, P<0.001)$, whereas Staphylococcus spp. were more frequently isolated from healthcare-related meningitis patients $(44.1 \%$ vs. $4.7 \%, P<0.001)$. Furthermore, L. monocytogenes was only isolated from community-acquired meningitis patients, whereas CoNS and Enterobacter spp. were only isolated from healthcare-related meningitis patients.

Antimicrobial susceptibility profiles. The susceptibility rates of $S$. pneumoniae to penicillin G, cefotaxime, and vancomycin were $33.3 \%, 40.0 \%$, and $100 \%$, respectively (Supplementary Table S1). For streptococci other than $S$. pneumoniae, the susceptibility rates to penicillin and vancomycin were $62.5 \%$ and $100 \%$, respectively. Among the S. aureus isolates, $85 \%$ were resistant to methicillin (oxacillin), but $100 \%$ were susceptible to vancomycin. S. epidermidis isolates showed similar profiles, with $90.5 \%$ resistant to methicillin (oxacillin), but $100 \%$ susceptible to vancomycin. The MDR rate of Staphylococcus spp. was $88.5 \%$. Among gram-negative bacilli, the susceptibility rates of $K$. pneumoniae to ceftriaxone, cefepime, and meropenem were $85.7 \%, 81.3 \%$, and $100 \%$, respectively (Supplementary Table S2). Extended-spectrum $\beta$-lactamase (ESBL) producers were found to account for $18.8 \%(3 / 16)$ of the K. pneumoniae isolates, all of which were from healthcare-related meningitis patients. The MDR rates of K. pneumoniae, Pseudomonas spp., Acinetobacter spp., and Enterobacter spp. were $25.0 \%, 18.2 \%, 44.4 \%$, and $56.3 \%$, respectively. Overall, bacterial isolates from healthcare-related meningi- 


\begin{tabular}{|c|c|c|c|c|}
\hline & $\begin{array}{l}\text { Total } \\
(n=161)\end{array}$ & $\begin{array}{l}\text { Community-acquired } \\
(\mathrm{n}=43)\end{array}$ & $\begin{array}{l}\text { Healthcare-related } \\
(\mathrm{n}=118)\end{array}$ & $P$ \\
\hline Complications & $39.1 \%$ & $65.1 \%$ & $29.7 \%$ & $<0.001$ \\
\hline Hydrocephalus & $19.9 \%$ & $16.3 \%$ & $21.2 \%$ & 0.490 \\
\hline Seizure & $13.7 \%$ & $18.6 \%$ & $11.9 \%$ & 0.271 \\
\hline Brain abscess & $8.7 \%$ & $14.0 \%$ & $6.8 \%$ & 0.204 \\
\hline Ischemic infarction & $5.6 \%$ & $18.6 \%$ & $0.8 \%$ & $<0.001$ \\
\hline Cerebral hemorrhage & $4.3 \%$ & $11.6 \%$ & $1.7 \%$ & 0.015 \\
\hline \multicolumn{5}{|l|}{ Outcomes } \\
\hline Length of stay, days & $76.7 \pm 97.6$ & $44.0 \pm 36.6$ & $88.6 \pm 109.6$ & $<0.001$ \\
\hline ICU admission & $52.8 \%$ & $51.2 \%$ & $53.4 \%$ & 0.802 \\
\hline mRS at discharge & $3.3 \pm 1.9$ & $3.1 \pm 1.9$ & $3.4 \pm 1.9$ & 0.289 \\
\hline mRS $0-3$ at discharge & $53.4 \%$ & $60.5 \%$ & $50.8 \%$ & 0.279 \\
\hline In-hospital mortality & $10.6 \%$ & $11.6 \%$ & $10.2 \%$ & 0.777 \\
\hline mRS at 3 months ${ }^{*}$ & $3.0 \pm 2.1$ & $2.5 \pm 2.2$ & $3.2 \pm 2.0$ & 0.075 \\
\hline mRS $0-3$ at 3 months & $60.6 \%$ & $68.4 \%$ & $57.7 \%$ & 0.247 \\
\hline Mortality at 3 months & $14.8 \%$ & $18.4 \%$ & $13.5 \%$ & 0.461 \\
\hline
\end{tabular}

Table 4. Complications and outcome of bacterial meningitis cases. Data are presented as mean $\pm \mathrm{SD}$ or $\%$. *3-month outcomes were available in 142 cases. Abbreviations: ICU, intensive care unit; mRS, modified Rankin Scale.

\begin{tabular}{|l|l|l|}
\hline \multirow{2}{*}{ Variables } & Unfavorable outcome & Mortality \\
\cline { 2 - 3 } & OR $(\mathbf{9 5 \%} \mathbf{C I})^{*}$ & OR (95\% CI) $\dagger$ \\
\hline Age, years & $1.03(1.00-1.06)$ & $1.02(0.98-1.06)$ \\
\hline Initial GCS score $\leq 8$ & $4.53(1.57-13.05)$ & $5.67(1.76-18.25)$ \\
\hline
\end{tabular}

Table 5. Multivariate analysis for unfavorable outcome and mortality at 3 months. Patients with premorbid mRS scores $0-2$ were analyzed $(n=124)$. ${ }^{\star}$ Sex, healthcare-related meningitis, concurrent infection, and high-sensitivity C-reactive protein $>10 \mathrm{mg} / \mathrm{dL}$ were included as covariates. $\dagger$ Sex, healthcare-related meningitis, immunocompromised status, thrombocytopenia $<100,000 / \mathrm{mm}^{3}$, and high-sensitivity C-reactive protein $>10 \mathrm{mg} / \mathrm{dL}$ were included as covariates. Abbreviations: OR, odds ratio; CI, confidence interval; GCS, Glasgow coma scale.

tis patients showed higher rates of MDR than those from community-acquired meningitis patients $(69.1 \%$ vs. $25.0 \%, P<0.001)$.

Complications and outcomes. The mean length of stay in the hospital was $76.7 \pm 97.6$ days, with $52.8 \%$ of patients treated in the intensive care unit. Mechanical ventilation was used in $36.0 \%$ of patients. Neurological complications occurred in $39.1 \%$ of patients and the most common complication was hydrocephalus (19.9\%), followed by seizures $(13.7 \%)$. Ischemic infarction and cerebral hemorrhage were more common in communityacquired patients than in healthcare-related patients $(P<0.001$ and 0.015 , respectively; Table 4$)$. Mortality rates at discharge and 3 months after discharge were $10.6 \%$ and $14.8 \%$, respectively. Mortality rates and mRS scores at discharge and 3 months after discharge did not differ between community-acquired and healthcare-related meningitis patients, although 3-month mRS scores in healthcare-related meningitis patients tended to be higher than those in community-acquired meningitis patients $(P=0.075)$.

We then assessed factors associated with unfavorable outcomes and mortality at 3 months in adult bacterial meningitis patients. Since 19 patients had no follow-up data at 3 months after discharge, we analyzed the outcome in 142 patients. In univariate analysis, an unfavorable outcome was associated with older age, neurological complications, concurrent infection, high hs-CRP levels, and an initial GCS score $\leq 8$. However, neither positive Gram staining results, the MDR status of isolates, nor immunocompromised status were associated with an unfavorable outcome (Supplementary Table S3). After adjusting for covariates, an unfavorable outcome was significantly associated with older age (odds ratio [OR] 1.03, 95\% confidence interval [CI] 1.00-1.06), neurological complications (OR 4.53, 95\% CI 1.57-13.05), and an initial GCS score $\leq 8$ (OR 19.71, 95\% CI 4.35-89.40; Table 5). Multivariate analysis for mortality at 3 months showed similar results with higher mortality rates associated with neurological complications (OR 5.67, 95\% CI 1.76-18.25) and an initial GCS score $\leq 8$ (OR 5.31, 95\% CI 1.47-19.11).

We additionally performed subgroup analysis on the unfavorable outcome for community-acquired and healthcare-related meningitis, respectively. In healthcare-related meningitis, older age (OR 1.07, 95\% CI 1.02-1.11), neurological complications (OR 4.13, 95\% CI 1.12-15.25), and an initial GCS score $\leq 8$ (OR 39.93, 
95\% CI 2.61-610.86) were associated with the unfavorable outcome, which is similar to the results from the total subjects. By contrast, an initial GCS score $\leq 8$ (OR 14.23, 95\% CI 1.82-111.31) only remained significantly associated with the unfavorable outcome in community-acquired meningitis (Table S4).

\section{Discussion}

In this study, we investigated clinical, laboratory, and microbiological profiles of adult bacterial meningitis. The composition of causative microorganisms was significantly different between community-acquired and healthcare-related meningitis. Streptococcus spp. accounted for $34.9 \%$ of community-acquired meningitis patients, whereas Staphylococcus spp. accounted for $44.1 \%$ of healthcare-related meningitis patients. At the species level, K. pneumoniae (25.6\%) was the most common causative bacterium in community-acquired meningitis, followed by S. pneumoniae (18.6\%) and L. monocytogenes (11.6\%). In healthcare-related meningitis patients, S. epidermidis $(17.8 \%)$ was the most common causative bacterium, followed by S. aureus (16.1\%). Mortality during hospitalization and 3 months after discharge were $10.6 \%$ and $14.8 \%$, respectively. Older age, any neurological complications, and severe mental deterioration at admission were significantly associated with unfavorable outcomes, which is consistent with the results of previous studies ${ }^{3,15}$.

A notable finding in this study was that $K$. pneumoniae was the most common pathogen in communityacquired meningitis patients. This is consistent with data from Taiwan showing that K. pneumoniae was the leading causative pathogen, accounting for $44.9 \%$ of spontaneous bacterial meningitis patients ${ }^{16}$. Although a high incidence of $K$. pneumoniae meningitis, together with a low incidence of $S$. pneumoniae meningitis has been reported in Taiwan in the 1980s and 1990s, the exact cause has not been determined ${ }^{17}$. Diabetes mellitus has been reported as a risk factor for community-acquired meningitis and liver abscesses caused by K. pneumo$n i a e^{18}$. In agreement with this, $54.5 \%(6 / 11)$ of community-acquired $K$. pneumoniae meningitis patients in our study had comorbid diabetes mellitus. However, this cannot explain the sudden change in the epidemiology of bacterial meningitis in Korea. An epidemiological study in Korean adults between 1998 and 2008 showed that K. pneumoniae was the third most common pathogen, but accounted for only $7.7 \%$ of community-acquired meningitis cases ${ }^{19}$. Selection bias due to a single-center study design may have contributed to our results. Nevertheless, our findings suggested that $K$. pneumoniae infection should be considered as a possible etiology of community-acquired bacterial meningitis, at least in the tertiary hospital setting. It also cannot be concluded whether the increase in K. pneumoniae meningitis is due to regional or racial influences in East Asian countries. Further epidemiological studies in other countries are needed to address this issue. All K. pneumoniae isolates from community-acquired meningitis patients were susceptible to cefotaxime and cefepime. ESBL-producing $K$. pneumoniae, all of which were isolated from healthcare-related meningitis patients, were resistant to ceftazidime and cefepime, but susceptible to imipenem and meropenem. These antimicrobial susceptibility profiles are consistent with previous studies ${ }^{20}$, suggesting that the third- and fourth-generation cephalosporins are appropriate for antibiotic therapy against community-acquired meningitis associated with K. pneumoniae, but carbapenems should be considered for the treatment of healthcare-related meningitis.

S. pneumoniae has traditionally been reported as the most common causative organism of communityacquired bacterial meningitis in adults ${ }^{3,21}$. However, $S$. pneumoniae only accounted for $18.6 \%$ of cases in our study, although it was the second most common species detected. The introduction of conjugate vaccines against S. pneumoniae is a possible explanation for the low frequency of pneumococcal meningitis. Pneumococcal vaccination significantly decreased the incidence of pneumococcal meningitis in children and adults, suggesting an indirect effect of vaccination through herd immunity ${ }^{22,23}$. The high rate of resistance of $S$. pneumoniae to penicillin and third-generation cephalosporins confirmed that antibiotics containing vancomycin are the appropriate empirical regimen for $S$. pneumoniae meningitis. The low frequency of $H$. influenzae meningitis in this study is also thought to be the result of $H$. influenzae type b vaccination ${ }^{6}$. In Korea, the incidence of $H$. influenzae meningitis in children has significantly decreased since $2001^{24}$, and in a previous study, $H$. influenzae was not isolated among 196 adult patients with community-acquired meningitis ${ }^{19}$.

L. monocytogenes was the third most common pathogen detected in our study population. It is well known that elderly individuals and immunocompromised patients are at a high risk of contracting L. monocytogenes meningitis ${ }^{25}$. In our study, the mean age of L. monocytogenes meningitis patients was 73.6 years (range, 66-83 years) and two (40\%) patients were immunocompromised. The two (40\%) immunocompromised patients died in hospital and the other two (40\%) were severely disabled (mRS score, 5) at 3 months, thus confirming the high mortality and morbidity rates previously reported for $L$. monocytogenes meningitis ${ }^{26}$.

The frequency of healthcare-related meningitis (73.3\%) was higher than that of community-acquired meningitis $(26.7 \%)$ in our study. These findings are likely to be caused by selecting bacterial meningitis cases from the tertiary hospital. Patients with community-acquired meningitis, especially those with mild severity, might have been treated at a lower level hospital rather than being transferred to this hospital. It is also possible that the epidemiological trend of decreasing frequencies of pneumococcal and meningococcal meningitis contributed to a decrease in the overall number of community-acquired meningitis and a relative increase in the proportion of healthcare-related meningitis ${ }^{10}$. However, it cannot be concluded in this study, and a nationwide epidemiological investigation is required to address this issue. The most common microorganisms causing healthcare-related bacterial meningitis were CoNS, S. aureus, and gram-negative bacilli, which were consistent with the results of previous studies ${ }^{1721}$. Staphylococcus spp. were mostly methicillin-resistant, but all were susceptible to vancomycin. Gram-negative bacilli showed moderate susceptibility to third- and fourth-generation cephalosporins, but high susceptibility to carbapenems. These antimicrobial susceptibility profiles support the use of vancomycin plus meropenem for the empirical treatment of healthcare-related meningitis ${ }^{27}$. Exceptionally, Acinetobacter spp. showed high resistance (44.4\%) to meropenem. Therefore, if the Acinetobacter isolates are resistant to carbapenems, colistin or polymyxin B should be administered ${ }^{28}$. 
There are several limitations of this study. Firstly, as a single-center study, our subjects may not represent the general population of adult bacterial meningitis patients in Korea. Recruitment from a tertiary hospital may have caused selection bias toward more severe cases. Moreover, although we collected data over a 10-year period, the sample size was relatively small, especially for community-acquired meningitis. Another limitation is the retrospective chart review design, which may have resulted in incomplete or inaccurate data collection. We first investigated the results of CSF examinations and cultures, and among probable cases and culture-positive cases, we reviewed the electrical medical records and selected those who met the definition of laboratory-confirmed bacterial meningitis. Nevertheless, we might have missed some cases, which is an inevitable limitation of the retrospective study. Furthermore, although we identified the clinical factors associated with unfavorable outcomes in bacterial meningitis patients, a causal relationship cannot be inferred from this study. Although we adjusted for the effect of the type of infection in the multivariate analysis, we cannot completely rule out the effect of neurosurgery itself on the unfavorable outcome.

In conclusion, we identified a spectrum of causative microorganisms for adult bacterial meningitis cases over a recent 10-year period. The increased proportion of K. pneumoniae infections and the decreased proportion of S. pneumoniae infections among community-acquired meningitis patients was particularly noteworthy. Our data on causative microorganisms and their antibiotic susceptibility profiles may help optimize determination of the appropriate empirical antimicrobial therapy for adult bacterial meningitis patients. However, further studies are required to confirm the changing epidemiology of causative pathogens and prognostic factors in adult bacterial meningitis.

\section{Data availability}

Deidentified data are available from the corresponding author upon reasonable request.

Received: 17 November 2020; Accepted: 1 March 2021

Published online: 16 March 2021

\section{References}

1. Liu, L. et al. Global, regional, and national causes of child mortality: an updated systematic analysis for 2010 with time trends since 2000. The Lancet 379, 2151-2161. https://doi.org/10.1016/s0140-6736(12)60560-1 (2012).

2. de Gans, J., van de Beek, D. \& European Dexamethasone in Adulthood Bacterial Meningitis Study Investigators. Dexamethasone in adults with bacterial meningitis. N. Engl. J. Med. 347, 1549-1556. https://doi.org/10.1056/NEJMoa021334 (2002).

3. van de Beek, D. et al. Clinical features and prognostic factors in adults with bacterial meningitis. N. Engl. J. Med. 351, 1849-1859. https://doi.org/10.1056/NEJMoa040845 (2004).

4. Auburtin, M. et al. Detrimental role of delayed antibiotic administration and penicillin-nonsusceptible strains in adult intensive care unit patients with pneumococcal meningitis: the PNEUMOREA prospective multicenter study. Crit. Care Med. 34, 2758-2765. https://doi.org/10.1097/01.ccm.0000239434.26669.65 (2006).

5. Tunkel, A. R. et al. Practice guidelines for the management of bacterial meningitis. Clin. Infect. Dis. 39, 1267-1284. https://doi. org/10.1086/425368 (2004).

6. Schuchat, A. et al. Bacterial meningitis in the United States in 1995 Active Surveillance Team. N. Engl. J. Med. 337, 970-976. https ://doi.org/10.1056/NEJM199710023371404 (1997).

7. Sigurdardottir, B., Bjornsson, O. M., Jonsdottir, K. E., Erlendsdottir, H. \& Gudmundsson, S. Acute bacterial meningitis in adults A 20-year overview. Arch. Intern. Med. 157, 425-430. https://doi.org/10.1001/archinte.1997.00440250077009 (1997).

8. Laxmi, S. \& Tunkel, A. R. Healthcare-associated bacterial meningitis. Curr. Infect. Dis. Rep. 13, 367-373. https://doi.org/10.1007/ s11908-011-0190-z (2011).

9. Thigpen, M. C. et al. Bacterial meningitis in the United States, 1998-2007. N. Engl. J. Med. 364, 2016-2025. https://doi.org/10.1056/ NEJMoa1005384 (2011).

10. Castelblanco, R. L., Lee, M. \& Hasbun, R. Epidemiology of bacterial meningitis in the USA from 1997 to 2010: a population-based observational study. Lancet. Infect. Dis 14, 813-819. https://doi.org/10.1016/s1473-3099(14)70805-9 (2014).

11. Song, J. H. et al. Spread of drug-resistant Streptococcus pneumoniae in Asian countries: Asian Network for Surveillance of Resistant Pathogens (ANSORP) Study. Clin. Infect. Dis. 28, 1206-1211. https://doi.org/10.1086/514783 (1999).

12. World Health Organization. Surveillance standards for vaccine-preventable diseases. 2nd edn, (World Health Organization, 2018)

13. Magiorakos, A. P. et al. Multidrug-resistant, extensively drug-resistant and pandrug-resistant bacteria: an international expert proposal for interim standard definitions for acquired resistance. Clin. Microbiol. Infect. 18, 268-281. https://doi.org/10.111 1/j.1469-0691.2011.03570.x (2012).

14. Richter, S. S. et al. Changing epidemiology of antimicrobial-resistant Streptococcus pneumoniae in the United States, 2004-2005. Clin. Infect. Dis. 48, e23-33. https://doi.org/10.1086/595857 (2009).

15. Flores-Cordero, J. M. et al. Acute community-acquired bacterial meningitis in adults admitted to the intensive care unit: clinical manifestations, management and prognostic factors. Intensive Care Med. 29, 1967-1973. https://doi.org/10.1007/s00134-0031935-4 (2003).

16. Chang, W. N. et al. Changing epidemiology of adult bacterial meningitis in southern taiwan: a hospital-based study. Infection 36, 15-22. https://doi.org/10.1007/s15010-007-7009-8 (2008).

17. Lu, C. H., Chang, W. N. \& Chang, H. W. Adult bacterial meningitis in Southern Taiwan: epidemiologic trend and prognostic factors. J. Neurol. Sci. 182, 36-44. https://doi.org/10.1016/s0022-510x(00)00445-7 (2000).

18. Ko, W.-C. et al. Community-acquired Klebsiella pneumoniae bacteremia: global differences in clinical patterns. Emerg. Infect. Dis. 8, 160-166. https://doi.org/10.3201/eid0802.010025 (2002).

19. Moon, S. Y. et al. Changing etiology of community-acquired bacterial meningitis in adults: a nationwide multicenter study in Korea. Eur. J. Clin. Microbiol. Infect. Dis. 29, 793-800. https://doi.org/10.1007/s10096-010-0929-8 (2010).

20. Ku, Y.-H. et al. Klebsiella pneumoniae Isolates from Meningitis: Epidemiology Virulence and Antibiotic Resistance. Sci. Rep. 7, 6634. https://doi.org/10.1038/s41598-017-06878-6 (2017).

21. Durand, M. L. et al. Acute bacterial meningitis in adults. A review of 493 episodes. N. Engl. J. Med. 328, 21-28. https://doi. org/10.1056/NEJM199301073280104 (1993).

22. Hsu, H. E. et al. Effect of pneumococcal conjugate vaccine on pneumococcal meningitis. N. Engl. J. Med. 360, 244-256. https:// doi.org/10.1056/NEJMoa0800836 (2009).

23. Tsai, C. J., Griffin, M. R., Nuorti, J. P. \& Grijalva, C. G. Changing epidemiology of pneumococcal meningitis after the introduction of pneumococcal conjugate vaccine in the United States. Clin. Infect. Dis. 46, 1664-1672. https://doi.org/10.1086/587897 (2008). 
24. Cho, H. K. et al. The causative organisms of bacterial meningitis in Korean children in 1996-2005. J. Korean Med. Sci. 25, 895-899. https://doi.org/10.3346/jkms.2010.25.6.895 (2010).

25. Brouwer, M. C., van de Beek, D., Heckenberg, S. G., Spanjaard, L. \& de Gans, J. Community-acquired Listeria monocytogenes meningitis in adults. Clin. Infect. Dis. 43, 1233-1238. https://doi.org/10.1086/508462 (2006).

26. Amaya-Villar, R. et al. Three-year multicenter surveillance of community-acquired Listeria monocytogenes meningitis in adults. BMC Infect. Dis. 10, 324. https://doi.org/10.1186/1471-2334-10-324 (2010).

27. Tunkel, A. R. et al. Infectious diseases society of america's clinical practice guidelines for healthcare-associated ventriculitis and meningitis. Clin. Infect. Dis. 64, e34-e65. https://doi.org/10.1093/cid/ciw861 (2017)

28. Kim, B.-N. et al. Management of meningitis due to antibiotic-resistant Acinetobacter species. Lancet. Infect. Dis 9, 245-255. https ://doi.org/10.1016/S1473-3099(09)70055-6 (2009).

\section{Acknowledgement}

The abstract of this study was published under the title "Clinical and Microbiological Characteristics of Bacterial Meningitis in Adults" at the 145th Annual Meeting of the American Neurological Association (https://doi. org/10.1002/ana.25865).

\section{Author contributions}

J.S.S., H.R.S., H.S.L., and K.C. contributed to the conception and design of the study; all authors contributed to the acquisition, analysis, or interpretation of data; J.S.S. and K.C. contributed to drafting a significant portion of the manuscript.

\section{Funding}

This work was supported by a research grant from Ildong Pharmaceutical, Co., Ltd, Seoul, South Korea (06-2019-1880).

\section{Competing interests}

The authors declare no competing interests.

\section{Additional information}

Supplementary Information The online version contains supplementary material available at https://doi. org/10.1038/s41598-021-85382-4.

Correspondence and requests for materials should be addressed to K.C.

Reprints and permissions information is available at www.nature.com/reprints.

Publisher's note Springer Nature remains neutral with regard to jurisdictional claims in published maps and institutional affiliations.

Open Access This article is licensed under a Creative Commons Attribution 4.0 International License, which permits use, sharing, adaptation, distribution and reproduction in any medium or format, as long as you give appropriate credit to the original author(s) and the source, provide a link to the Creative Commons licence, and indicate if changes were made. The images or other third party material in this article are included in the article's Creative Commons licence, unless indicated otherwise in a credit line to the material. If material is not included in the article's Creative Commons licence and your intended use is not permitted by statutory regulation or exceeds the permitted use, you will need to obtain permission directly from the copyright holder. To view a copy of this licence, visit http://creativecommons.org/licenses/by/4.0/.

(C) The Author(s) 2021 